\title{
Intrarenal arteriosclerosis and impairment of kidney function in NIDDM subjects
}

\author{
D. Boeri ${ }^{1}$, L.E. Derchi ${ }^{3}$, C.Martinoli ${ }^{3}$, G.Simoni ${ }^{4}$, L.Sampietro ${ }^{1}$, D. Storace ${ }^{1}$, L.Ponte ${ }^{1}$, C. Calvi $^{2}$, M. Repetto ${ }^{2}$, \\ C. Robaudo ${ }^{2}$, M.Maiello ${ }^{1}$ \\ ${ }^{1}$ Cattedra di Clinica Medica RR, Dipartimento di Medicina Interna, Università di Genova, Genova, Italy \\ ${ }^{2}$ Cattedra di Nefrologia, Dipartimento di Medicina Interna, Università di Genova, Genova, Italy \\ ${ }^{3}$ Istituto di Radiologia, Università di Genova, Genova, Italy \\ ${ }^{4}$ Cattedra di Clinica Chirurgica B, Università di Genova, Genova, Italy
}

Summary It is currently under debate whether the pathogenesis of end-stage renal failure in non-insulindependent diabetes mellitus (NIDDM) is a consequence of microangiopathy alone. The aim of this study was to investigate intrarenal arteriosclerosis and its correlation with kidney function in NIDDM. In 36 diabetic subjects, and in 10 age- and sex-matched healthy control subjects we measured kidney volume and resistive index of the interlobar arteries by duplex Doppler ultrasonography. Clinical and metabolic parameters, renal function and vascular sequelae of the disease were also evaluated. In diabetic subjects resistive index (median 0.72, range 0.54-0.79 ) was higher than in control subjects (median 0.62 , range $0.57--0.66)(2 p<0.002)$. Kidney volume and resistive index correlated with age $(p<0.004)$, body mass index $(p<0.001)$, mean blood pressure $(p<0.001)$, total and LDL cholesterol $(p<0.01)$ and creatinine clearance $(p<0.001$ and $<0.01$, respectively). Kidney volume also correlated with $\mathrm{HbA}_{1}$ $(p<0.01)$ and resistive index with uric acid $(p<0.01)$. Lower body macroangiopathy was associated with increased resistive index and reduced kidney volume $(2 p<0.05)$, while upper body macroangiopathy and microangiopathy were not. Our data suggest that macroangiopathy rather than microangiopathy is mainly responsible for impairment of kidney function in NIDDM. The resistive index of interlobar arteries seems to be a reliable marker of intrarenal arteriosclerosis and can be used as a non-invasive, easily available parameter of its evolution. [Diabetologia (1998) 41: 121--124]

Keywords Non-insulin-dependent diabetes mellitus, duplex Doppler ultrasonography, kidney, arteriosclerosis, albuminuria.
In the last few years western countries have experienced a dramatic change in the prognosis of renal involvement in non-insulin-dependent-diabetes mellitus (NIDDM), that was previously considered benign [1]. The 1994 United States Renal Data System Report indicated that $35 \%$ of patients entering renal replacement therapy programmes were diabetic. While the proportion of insulin-dependent diabetic

Received: 27 August 1997 and in revised form:

21 October 1997

Corresponding author: Dr. D. Boeri, Cattedra di Clinica Medica RR, Dipartimento di Medicina Interna, Viale Benedetto XV n. 6, 16132 Genova, Italy

Abbreviations: A/CR, Albumin to creatinine ratio; DDU, duplex Doppler ultrasonography; ESRF, end-stage renal failure; IDDM, insulin-dependent diabetes mellitus; MBP, mean blood pressure; NIDDM, non-insulin-dependent diabetes mellitus; $\mathrm{RI}$, resistive index.
(IDDM) patients developing end-stage renal failure (ESRF) remains stable over time, the prevalence of NIDDM is increasing [2], probably because of its increased incidence, due to the general aging of western populations. The fact that these patients also survive ischaemic heart disease more than in the past may also contribute to a higher incidence of ESRF [1].

Diabetic nephropathy, characterized by persistent proteinuria, is a well-known microvascular complication of both IDDM and NIDDM. However, in NIDDM albuminuria does not correlate with impairment of renal function and, in turn, ESRF is not associated with albuminuric nephropathy in up to $54 \%$ of patients [1].

It is thus under scrutiny whether ESRF in diabetes, and particularly in NIDDM, can be considered a consequence of microangiopathy alone [3].

Signs and symptoms of renal disease in a diabetic patient do not necessarily reflect the typical diffuse 
Table 1. Clinical and metabolic characteristics of subjects studied

\begin{tabular}{|c|c|c|c|}
\hline & NIDDM & & Control \\
\hline Subjects (male/female) & $36(14 / 22)$ & & $10(3 / 7)$ \\
\hline Age (years) & $61 \pm 7$ & N.S. & $59 \pm 6$ \\
\hline Known duration of diabetes (years) & $12 \pm 8$ & & \\
\hline Body mass index $(\%)$ & $29 \pm 5$ & N.S. & $28 \pm 6$ \\
\hline Mean blood pressure (mm Hg) & $108 \pm 9$ & N.S. & $103 \pm 7$ \\
\hline Smokers & $3 / 36$ & N.S. & $1 / 10$ \\
\hline Fasting blood glucose (mmol/l) & $10.9 \pm 4.5$ & $2 p<0.05$ & $4.8 \pm 0.7$ \\
\hline $\mathrm{HbA}_{1}(\%)$ & $6.35 \pm 1.8$ & $2 p<0.01$ & $3.9 \pm 1.7$ \\
\hline Total cholesterol (mmol/l) & $61.8 \pm 11.6$ & N.S. & $55 \pm 6$ \\
\hline HDL-Cholesterol (mmol/1) & $11.9 \pm 3.9$ & N.S. & $12 \pm 1.3$ \\
\hline LDL-Cholesterol (mmol/l) & $31.0 \pm 12.7$ & N.S. & $34 \pm 4.7$ \\
\hline Triglycerides (mmol/l) & $22.5 \pm 9.8$ & N.S. & $19.3 \pm 3.4$ \\
\hline Uric acid $(\mu \mathrm{mol} / 1)$ & $299 \pm 89$ & N.S. & $268 \pm 53.5$ \\
\hline Creatinine $(\mu \mathrm{mol} / \mathrm{l})$ & $89.7 \pm 28.3$ & N.S. & $70.7 \pm 5.3$ \\
\hline Creatinine clearance $(\mathrm{ml} / \mathrm{s})$ & $1.71 \pm 0.9$ & N.S. & $1.75 \pm 0.5$ \\
\hline Albumin/creatinine ratio & $5.5 \pm 9$ & $2 p<0.01$ & $1.7 \pm 0.8$ \\
\hline Kidney volume (ml) & $253(153--340)$ & N.S. & $262(219--282)$ \\
\hline RI & $0.72(0.54--0.79)$ & $2 p<0.002$ & $0.62(0.57--0.66)$ \\
\hline
\end{tabular}

Data are mean \pm SD or median (range)

or nodular diabetic glomerulosclerosis. Arteriosclerosis, tubulo-interstitial infections, or glomerulopathies of diverse origins can be solely responsible for the renal involvement in up to $24 \%$ of NIDDM patients with ESRF [1] or can be associated with the typical diabetic albuminuric syndrome, as demonstrated by morphology $[1,4]$.

Histological evidence of arteriosclerosis is commonly found in kidneys of patients with NIDDM [4], but in the absence of reliable non-invasive techniques to measure large vessel pathology in the kidney, prevalence, evolution and the relevance of the vascular abnormalities to renal function have not been defined.

The recent availability of duplex Doppler ultrasonography (DDU) has provided a rapid and non-invasive method with which to study the characteristics of intrarenal blood flow [5]. The method permits the evaluation of changes in arterial impedance, as defined by the intrarenal resistive index (RI), which is increased in vascular and tubulointerstitial pathologies, while it is not influenced by pure glomerular disease [6].

The aim of this study was to evaluate the relevance of intrarenal macroangiopathy in the development and/or the evolution of renal involvement in NIDDM.

\section{Subjects and methods}

Thirty-six NIDDM subjects entered the study (Table 1). All patients were treated with diet, supplemented by oral hypoglycaemic agents in 27 patients; 5 subjects were insulin-treated. Nine subjects were treated for hypertension (2 patients received angiotensin-converting enzyme inhibitors, 2 calcium-entry blockers, 2 beta-adrenergic blockers, 3 alpha- 1 receptor blockers). We excluded patients with severely impaired renal function (serum creatinine level above $177 \mu \mathrm{mol} / \mathrm{l}$ ) or signs and symptoms of renal interstitial and tubular diseases characterized by 1) negative clinical history for acute and chronic urinary tract infections; 2) immunologic, urologic, and metabolic diseases; 3) sickle cell disease; 4) hereditary diseases and/or drugs known to affect interstitial and tubular functions; 5) negative urine microscopy and culture analysis; 6) normal urine osmolarity.

The presence of renal artery stenosis was excluded on the basis of clinical findings (absence of drug-resistant or malignant hypertension, hyponatriaemic hypertensive syndrome) and of indirect ultrasonographic parameters. A group of 10 healthy volunteers, matched for age, sex and body mass index, was used as control. Informed consent was obtained from all subjects; the study was performed in accordance with the principles of the 1975 Declaration of Helsinki. Blood pressure was recorded at the time of the DDU examination and reported as mean blood pressure:

$\mathrm{MBP}=$ systolic blood pressure + $\frac{\text { systolic-diastolic blood pressure }}{3}$

Blood and urine samples, as summarized in Table 1, were obtained within 1 week from the ultrasonographic examination. Vascular abnormalities were evaluated as follows. Diabetic retinopathy was detected by direct ophthalmoscopy and/or fluorangiography. The patients were divided into two groups, according to the absence of retinopathic alterations or the presence of retinopathy, either background and/or proliferative. Diabetic nephropathy was defined according to the albumin-to-creatinine ratio on three urinary samples (A/CR, albu$\mathrm{min} /$ creatinine ratio). (Negative: $<2.38$ for males, $<2.96$ for females; positive: $>2.38$ for males, $>2.96$ for females). Serum and urinary creatinine levels were determined by the Jaffé reaction. Urinary albumin concentration was determined by a commercial radioimmunoassay kit (Sclavo, Cinisello Balsamo, Italy). Macroangiopathy was assessed by conventional Doppler ultrasonography of extracranial arteries (upper body macroangiopathy), and of posterior tibial and dorsalis pedis arteries (lower body macroangiopathy) (Doppler CW Sonicaid Vasoflow 2, $8 \mathrm{MHz}$, Chichester, West Sussex, United Kingdom). The presence of pathologic changes of the arterial wall was inferred from the characteristics of the Doppler waveform (negative: normal vessel wall, positive: arteriosclerotic changes). DDU of the renal interlobar arteries was performed with a (Idea AU4, Esaote Biomedica (Genova, Italy) with a 
Table 2. Kidney volume and resistive index (RI)

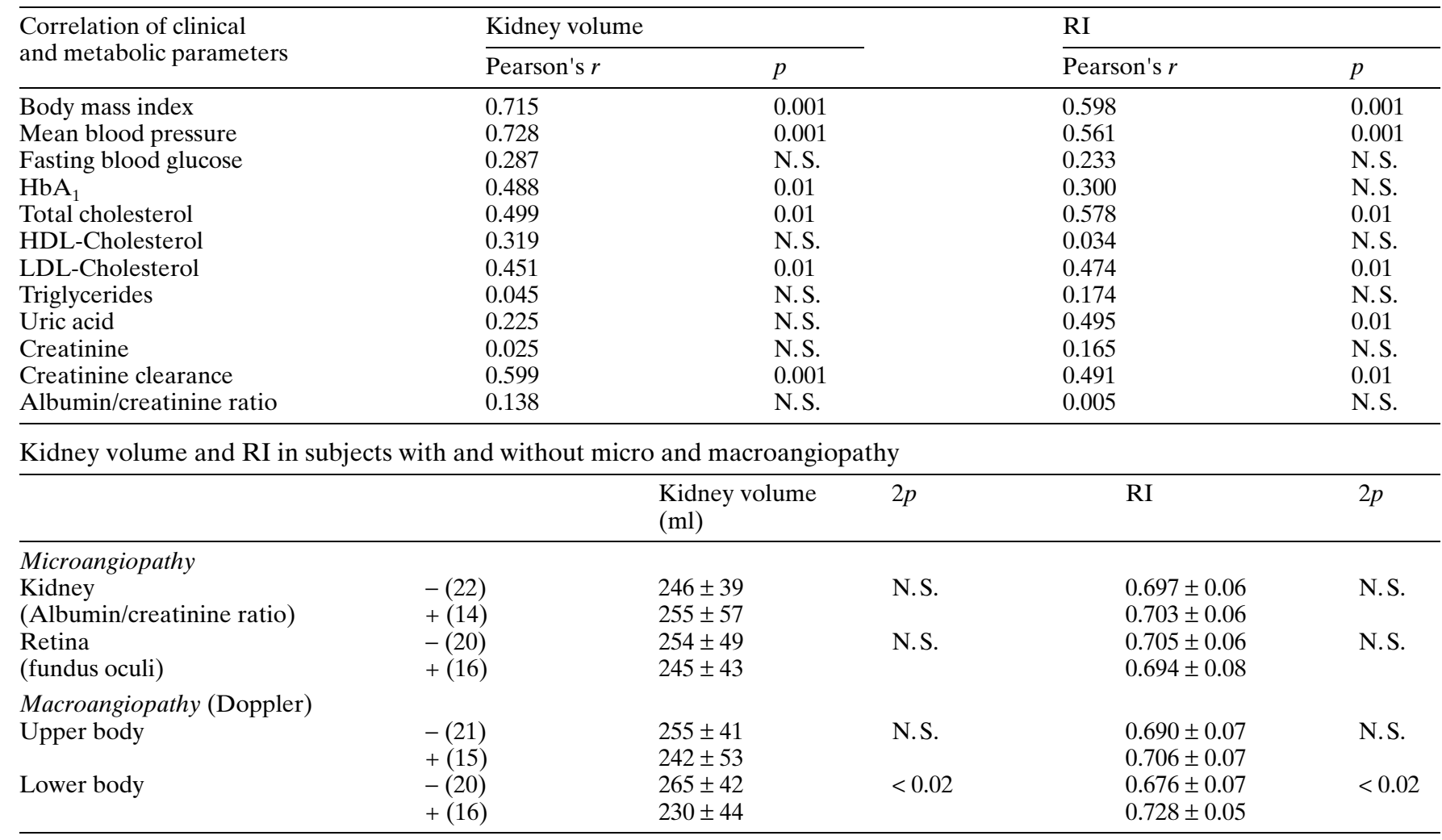

curved array transducer used at $3.5 \mathrm{MHz}$ for imaging and $2.5 \mathrm{MHz}$ for Doppler analysis. Renal volume was calculated by a three-dimensional ellipsoid formula, derived from the cranio-caudal, mediolateral and anteroposterior diameters [7]. The RI was calculated by the formula:

peak systolic value - end diastolic value peak systolic value

Three different measurements were obtained for each kidney in different portions of the organ (upper, middle and lower pole); the result represents the mean of the six measurements. The coefficient of variation was $4 \pm 1.4 \%$. Values of RI higher than 0.7 were considered pathologic [5].

Statistical analysis. Data are presented as mean \pm SD or median and range, where appropriate. Statistical analysis was performed with Student's $t$-test and/or Mann-Whitney test, multiple regression analysis and Pearson's correlation test. Since the multiple regression analysis indicated the age of the subjects as the strongest influencing factor for both kidney volume (partial $F: 9.376, p<0.004$ ) and RI (partial $F: 23.011, p<0.0001$ ) the correlation coefficient was calculated after normalization for age.

\section{Results}

Clinical and metabolic characteristics of the subjects are summarized in Table 1. The renal volume in diabetic patients was slightly but not significantly lower than in control subjects (Table 1). It correlated with age, body mass index, MBP, $\mathrm{HbA}_{1}$, total and LDL cholesterol and creatinine clearance (Table 2).

Renal RI in the diabetic patients was significantly higher than in control subjects (Table 1 ). In 22 out of 36 diabetic subjects $(62 \%)$ the RI was higher than 0.7 . RI correlated with age, body mass index, MBP, uric acid, total and LDL cholesterol and creatinine clearance (Table 2).

Sixteen of the 36 diabetic patients presented Doppler examination positive for macroangiopathy of the lower limbs, while the remaining 20 were negative. The macroangiopathy-positive subjects showed significantly higher RI $(0.73 \pm 0.05$ vs $0.68 \pm 0.07,2 p<0.05)$ and reduced kidney volume $(230 \pm 44$ vs $265 \pm 43,2 p<0.05)$. The presence of macroangiopathy of the upper body as well as of retinal and renal microangiopathy was not associated with differences of renal volume or RI (Table 2).

Microalbuminuria (A/CR) was not correlated with renal volume, RI, or with any metabolic parameter.

\section{Discussion}

The kidneys of diabetic subjects present at least two typical morphological features. The first is an early increase in volume, which accompanies the increased glomerular filtration rate, typically reported in 
IDDM but also present in NIDDM. The second is a late evolution of reduction in kidney volume that is often reported in NIDDM and can be considered a feature of ischaemic nephropathy [1].

In our diabetic subjects, although slightly reduced on average, the kidney volume was not significantly different from that of control subjects. Early nephromegaly could have been missed in this population, since the average known duration of the disease was 12 years, while the progressive reduction in volume was not yet evident since kidney function ranged from normal to initial impairment. Nevertheless, the correlation between kidney volume and creatinine clearance indicates that the reduction of kidney volume proceeds in parallel with the impairment of renal function. Furthermore, the correlation between kidney volume and macroangiopathy risk factors -- body mass index, MBP, total and LDL cholesterol -- strongly suggests the ischaemic nature of the initial impairment in renal function in some of our diabetic subjects.

Alterations of the intrarenal RI have been demonstrated both in diabetic patients with detectable nephropathy $[8,9]$, and in patients with normal renal function [7].

Increased RI has been related to vascular, interstitial and tubular nephropathies. The selection criteria for diabetic patients entering this study excluded associated interstitial nephropathies and/or tubular diseases.

The correlations between RI and macroangiopathy risk factors such as body mass index, MBP, total and LDL cholesterol, strongly suggest that intrarenal arteriosclerosis is responsible for the increased RI. This hypothesis is supported by the observation that $\mathrm{RI}$ is significantly higher in subjects with macroangiopathy of the lower limbs.

RI correlates with creatinine clearance, indicating that an increase of intrarenal vascular impedance proceeds in parallel with the impairment of renal function.

These correlations are of particular relevance, since they are obtained in a cross-section study and in a group of subjects with kidney function ranging from normal to mildly impaired.

The increase of RI is not related to microalbuminuria, indicating, as already described, that the intrarenal impedance is not influenced by glomerulopathy [6].

In accordance with previous studies, microalbuminuria $(\mathrm{A} / \mathrm{CR})$ did not correlate with creatinine clearance, indicating that the initial impairment of kidney function in some of these subjects, is not due to the diabetic microangiopathy.

Previous studies addressed the issue of possible relations between diabetic nephropathy and RI, and found that this parameter was mainly increased in patients with impaired renal function, while its relations with microalbuminuria or overt proteinuria are under debate [7--9].
Recently, Lam et al. [10] demonstrated that cholesterol-lowering therapy retarded the progression of nephropathy in NIDDM, reducing the rate of impairment of kidney function, without affecting the albumin excretion rate [10]. This finding can also be interpreted as evidence for a major role for macroangiopathy in the evolution towards ESRF in NIDDM.

In our study, an RI higher than normal, suggestive of initial arteriosclerosis, was demonstrated in $62 \%$ of the diabetic subjects. The true incidence of intrarenal arteriosclerosis can be underestimated when invasive techniques such as arteriography or biopsy are used. In fact, arteriography visualizes only the most advanced alterations of intrarenal arteries, while renal biopsy identifies very initial lesions in a limited area that can be unrepresentative of the whole organ. Among the parameters obtained by DDU, RI is considered the most consistent and reproducible [5]. In the present study the coefficient of variation of RI -six measurements in different areas of both kidneys for each subject -- was very small $(4 \pm 1.4 \%)$. The consistency of the measurements indicates that RI allows a global evaluation of intrarenal vascular impedance. This non-invasive and easily available parameter will be of value in following the evolution of intrarenal arteriosclerosis in the individual patient and in longitudinal studies.

Acknowledgements. This paper was in part supported by grants from Ministero dell' Università e della Ricerca Scientifica e Tecnologica (MURST $60 \% 1993$ and 1996) and from Comitato Nazionale della Ricerca (CNR96.03723.CT14), Italy.

\section{References}

1. Ritz E, Stefanski A (1996) Diabetic nephropathy in type II diabetes. Am J Kidney Diseases 27 (2): 167--174

2. The United States Renal Data System: USRDS (1994) Annual Data Report. The National Institutes of Health, National Institute of Diabetes and Digestive and Kidney Diseases, Bethesda, MD

3. Raine AEG, Bilous RW (1996) End-stage renal disease in NIDDM: a consequence of microangiopathy alone? Diabetologia 39: 1663-1675

4. Cordonnier D (1996) Glomerular involvement in type II diabetes -is it all diabetic glomerulosclerosis? Nephrol Dial Transplant 11: 936--938

5. Knapp R, Plotzeneder A, Frauscher F et al. (1995) Variability of Doppler parameters in the healthy kidney. J Ultrasound Med 14: 427--429

6. Platt JF, Ellis JH, Rubin JM, DiPietro MA, Sedman AB (1990) Intrarenal arterial Doppler sonography in patients with nonobstructive renal disease: correlation of resistive index with biopsy findings. AJR 154: 1223--1227

7. Derchi LE, Martinoli C, Saffiotti S, Pontremoli R, De Micheli A, Bordone C (1994) Ultrasonographic imaging and Doppler analysis of renal changes in non-insulin-dependent diabetes mellitus. Acad Radiol 1: 100--105

8. Platt JF, Rubin JM, Ellis JH (1994) Diabetic nephropathy: evaluation with renal Doppler US. Radiology 190: 343--346

9. Brkljacic B, Mrzljak V, Drinkovic I, Soldo D, Sabljar-Matovinovic M, Hebrang A (1994) Renal vascular resistance in diabetic nephropathy: Duplex Doppler US evaluation. Radiology 192: 549--554

10. Lam KS, Cheng IPK, Janus ED, Pang RWC (1995) Cholesterollowering therapy may retard the progression of diabetic nephropathy. Diabetologia 38: 604--609 